\title{
Discovery of very high energy gamma-ray emission coincident with molecular clouds in the W 28 (G6.4-0.1) field ${ }^{\star}$
}

F. Aharonian ${ }^{1,13}$, A. G. Akhperjanian ${ }^{2}$, A. R. Bazer-Bachi ${ }^{3}$, B. Behera ${ }^{14}$, M. Beilicke ${ }^{4}$, W. Benbow ${ }^{1}$, D. Berge ${ }^{1, \star \star}$, K. Bernlöhr ${ }^{1,5}$, C. Boisson ${ }^{6}$ O. Bolz ${ }^{1}$, V. Borrel ${ }^{3}$, I. Braun ${ }^{1}$, E. Brion ${ }^{7}$, A. M. Brown ${ }^{8}$, R. Bühler ${ }^{1}$, T. Bulik ${ }^{24}$, I. Büsching, ${ }^{9}$, T. Boutelier ${ }^{17}$, S. Carrigan ${ }^{1}$, P. M. Chadwick ${ }^{8}$, L.-M. Chounet ${ }^{10}$, A. C. Clapson $^{1}$, G. Coignet $^{11}$, R. Cornils ${ }^{4}$, L. Costamante ${ }^{1,25}$, B. Degrange ${ }^{10}$, H. J. Dickinson ${ }^{8}$, A. Djannati-Atai ${ }^{12}$, W. Domainko ${ }^{1}$, L. O'C. Drury ${ }^{13}$, G. Dubus ${ }^{10}$, J. Dyks ${ }^{24}$, K. Egberts ${ }^{1}$, D. Emmanoulopoulos ${ }^{14}$, P. Espigat ${ }^{12}$, C. Farnier ${ }^{15}$, F. Feinstein ${ }^{15}$, A. Fiasson ${ }^{15}$, A. Förster ${ }^{1}$, G. Fontaine ${ }^{10}$, Y. Fukuii ${ }^{26}$, Seb. Funk ${ }^{5}$, S. Funk ${ }^{1}$, M. Füßling ${ }^{5}$, Y. A. Gallant ${ }^{15}$, B. Giebels ${ }^{10}$,

J. F. Glicenstein ${ }^{7}$, B. Glück ${ }^{16}$, P. Goret ${ }^{7}$, C. Hadjichristidis ${ }^{8}$, D. Hauser ${ }^{1}$, M. Hauser ${ }^{14}$, G. Heinzelmann ${ }^{4}$, G. Henri ${ }^{17}$, G. Hermann ${ }^{1}$, J. A. Hinton ${ }^{1,14, \star \star \star}$, A. Hoffmann ${ }^{18}$, W. Hofmann ${ }^{1}$, M. Holleran ${ }^{9}$, S. Hoppe ${ }^{1}$, D. Horns ${ }^{18}$, A. Jacholkowska ${ }^{15}$, O. C. de Jager ${ }^{9}$, E. Kendziorra ${ }^{18}$, M. Kerschhaggl ${ }^{5}$, B. Khélifi ${ }^{10,1}$, Nu. Komin ${ }^{15}$, K. Kosack ${ }^{1}$, G. Lamanna ${ }^{11}$, I. J. Latham ${ }^{8}$, R. Le Gallou ${ }^{8}$, A. Lemière ${ }^{12}$, M. Lemoine-Goumard ${ }^{10}$, J.-P. Lenain ${ }^{6}$, T. Lohse ${ }^{5}$, J. M. Martin ${ }^{6}$, O. Martineau-Huynh ${ }^{19}$, A. Marcowith ${ }^{15}$, C. Masterson ${ }^{13}$, G. Maurin ${ }^{12}$, T. J. L. McComb ${ }^{8}$, R. Moderski ${ }^{24}$, Y. Moriguchi ${ }^{26}$, E. Moulin ${ }^{15,7}$, M. de Naurois ${ }^{19}$, D. Nedbal ${ }^{20}$, S. J. Nolan ${ }^{8}$, J.-P. Olive ${ }^{3}$, K. J. Orford ${ }^{8}$, J. L. Osborne ${ }^{8}$, M. Ostrowski ${ }^{23}$, M. Panter ${ }^{1}$, G. Pedaletti ${ }^{14}$, G. Pelletier ${ }^{17}$, P.-O. Petrucci ${ }^{17}$, S. Pita ${ }^{12}$, G. Pühlhofer ${ }^{14}$, M. Punch ${ }^{12}$, S. Ranchon ${ }^{11}$, B. C. Raubenheimer ${ }^{9}$, M. Raue ${ }^{4}$, S. M. Rayner ${ }^{8}$, O. Reimer ${ }^{\dagger}$, M. Renaud ${ }^{1}$, J. Ripken ${ }^{4}$, L. Rob ${ }^{20}$, L. Rolland ${ }^{7}$, S. Rosier-Lees ${ }^{11}$, G. Rowell ${ }^{1, \ddagger}$, B. Rudak ${ }^{24}$, J. Ruppel ${ }^{21}$, V. Sahakian ${ }^{2}$, A. Santangelo ${ }^{18}$, L. Saugé ${ }^{17}$, S. Schlenker ${ }^{5}$, R. Schlickeiser ${ }^{21}$, R. Schröder ${ }^{21}$, U. Schwanke ${ }^{5}$, S. Schwarzburg ${ }^{18}$, S. Schwemmer ${ }^{14}$,

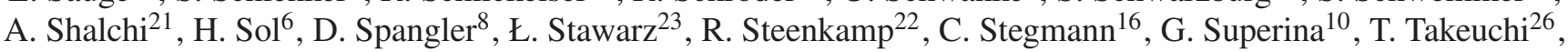
P. H. Tam ${ }^{14}$, J.-P. Tavernet ${ }^{19}$, R. Terrier ${ }^{12}$, C. van Eldik $^{1}$, G. Vasileiadis ${ }^{15}$, C. Venter ${ }^{9}$, J. P. Vialle ${ }^{11}$, P. Vincent ${ }^{19}$, M. Vivier ${ }^{7}$, H. J. Völk ${ }^{1}$, F. Volpe ${ }^{10}$, S. J. Wagner ${ }^{14}$, and M. Ward ${ }^{8}$

(Affiliations can be found after the references)

Received 1 May 2007 / Accepted 16 January 2008

\section{ABSTRACT}

Aims. Observations of shell-type supernova remnants (SNRs) in the $\mathrm{GeV}$ to multi-TeV $\gamma$-ray band, coupled with those at millimetre radio wavelengths, are motivated by the search for cosmic-ray accelerators in our Galaxy. The old-age mixed-morphology SNR W 28 (distance $\sim 2 \mathrm{kpc}$ ) is a prime target due to its interaction with molecular clouds along its northeastern boundary and other clouds situated nearby.

Methods. We observed the W 28 field (for $\sim 40 \mathrm{~h}$ ) at very high energy (VHE) $\gamma$-ray energies $(E>0.1 \mathrm{TeV})$ with the HESS. Cherenkov telescopes. A reanalysis of EGRET $E>100 \mathrm{MeV}$ data was also undertaken. Results from the NANTEN $4 \mathrm{~m}$ telescope Galactic plane survey and other $\mathrm{CO}$ observations were used to study molecular clouds.

Results. We have discovered VHE $\gamma$-ray emission (HESS J1801-233) coincident with the northeastern boundary of W 28 and a complex of sources (HESS J1800-240A, B and C) $\sim 5^{\circ}$ south of W 28 in the Galactic disc. The EGRET source (GRO J1801-2320) is centred on HESS J1801-233 but may also be related to HESS J1800-240 given the large EGRET point spread function. The VHE differential photon spectra are well fit by pure power laws with indices $\Gamma \sim 2.3$ to 2.7. The spectral indices of HESS J1800-240A, B, and C are consistent within statistical errors. All VHE sources are $\sim 10^{\prime}$ in intrinsic radius except for HESS J1800-240C, which appears pointlike. The NANTEN ${ }^{12} \mathrm{CO}(J=1-0)$ data reveal molecular clouds positionally associating with the VHE emission, spanning a $\sim 15 \mathrm{~km} \mathrm{~s}^{-1}$ range in local standard of rest velocity.

Conclusions. The VHE/molecular cloud association could indicate a hadronic origin for HESS J1801-233 and HESS J1800-240, and several cloud components in projection may contribute to the VHE emission. The clouds have components covering a broad velocity range encompassing the distance estimates for W $28(\sim 2 \mathrm{kpc})$ and extending up to $\sim 4 \mathrm{kpc}$. Assuming hadronic origin and distances of 2 and $4 \mathrm{kpc}$ for cloud components, the required cosmic-ray density enhancement factors (with respect to the solar value) are in the range $\sim 10$ to $\sim 30$. If situated at $2 \mathrm{kpc}$ distance, such cosmic-ray densities may be supplied by SNRs like W 28. Additionally and/or alternatively, particle acceleration may come from several catalogued SNRs and SNR candidates, the energetic ultra compact HII region W 28A2, and the HII regions M 8 and M 20, along with their associated open clusters. Further sub-mm observations would be recommended to probe in detail the dynamics of the molecular clouds at velocites $>10 \mathrm{~km} \mathrm{~s}^{-1}$ and their possible connection to $\mathrm{W} 28$.

Key words. gamma rays: observations

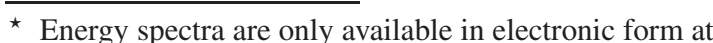
http: //www . aanda.org

$\star \star$ Now at CERN, Geneva, Switzerland.

$\star \star \star$ Now at School of Physics \& Astronomy, University of Leeds, Leeds LS2 9JT, UK.

\footnotetext{
Now at Stanford University, HEPL \& KIPAC, Stanford, CA 943054085, USA.

\# Now at School of Chemistry \& Physics, University of Adelaide, Adelaide 5005, Australia.
} 


\section{Introduction: W 28 and surroundings}

The study of shell-type supernova remnants (SNRs) at $\gamma$-ray energies is motivated by the long-held idea that they are the dominant sites of hadronic Galactic cosmic-ray (CR) acceleration to energies approaching the knee $\left(\sim 10^{15} \mathrm{eV}\right)$ (e.g. Ginzburg \& Syrovatskii 1964; Blandford \& Eichler 1987). CRs (hadrons and electrons) are injected into the SNR shock front, and are then accelerated via the diffusive shock acceleration (DSA) process (for a review see Drury 1983). Subsequent $\gamma$-ray production from the interaction of these CRs with ambient matter and/or electromagnetic fields is a tracer of such non-thermal particle acceleration, and establishing the hadronic or electronic nature of the parent CRs in any $\gamma$-ray source remains a key issue. Two SNRs, RX J1713.7-3946 and RX J0852.0-4622, have so far established shell-like morphology in VHE $\gamma$-rays (Aharonian et al. 2004a, 2005c, 2006b, 2007a, 2007b), with spectra extending to $20 \mathrm{TeV}$ and beyond. In particular for RX J1713.7-3946, particle acceleration up to at least $100 \mathrm{TeV}$ is inferred from the HESS observations. Although a hadronic origin of the VHE $\gamma$-ray emission is highly likely in the above cases (Aharonian et al. 2006b, 2007b; Berezhko \& Völk 2006; Berezhko et al. 2007), an electronic origin is not ruled out.

Disentangling the electronic and hadronic components in TeV SNRs may be made easier by studying: (1) SNR $\gamma$-ray spectra well beyond $\sim 10 \mathrm{TeV}$, an energy regime where electrons suffer strong radiative energy losses and due to Klein-Nishina effects the resulting inverse-Compton spectra tend to show a cut-off; (2) older SNRs (age approaching $10^{5} \mathrm{yr}$ ) in which accelerated electrons have lost much of their energy through radiative cooling and do not reach multi-TeV energies; (3) SNRs interacting with adjacent molecular clouds of very high densities $n>10^{3} \mathrm{~cm}^{-3}$. It is the latter regard especially (and to a certain degree the second) which makes the SNR W 28 (G6.4-0.1) an attractive target for VHE $\gamma$-ray studies. In this paper we outline the discovery of VHE $\gamma$-ray emission from several sites in the W 28 field and briefly discuss their relationship with molecular clouds, W 28, and other potential particle accelerators in the region.

W 28 (G6.4-0.1) is a mixed-morphology SNR, with dimensions $50^{\prime} \times 45^{\prime}$ and an estimated distance between 1.8 and $3.3 \mathrm{kpc}$ (e.g. Goudis 1976; Lozinskaya 1981). It is an old-age SNR (age 35000 to $150000 \mathrm{yr}$; e.g. Kaspi et al. 1993), thought to have entered its radiative phase of evolution (e.g. Lozinskaya 1981) in which much of its CRs have escaped into the surrounding interstellar medium (ISM). We note also that the evolutionary status (Sedov and/or radiative) of shell-type SNRs may depend on the density of their surroundings (see e.g. Blondin et al. 1998).

W 28 is distinguished by its interaction with a molecular cloud (Wootten 1981) along its north and northeastern boundaries. This interaction is traced by the high concentration of $1720 \mathrm{MHz} O \mathrm{OH}$ masers (Frail et al. 1994; Claussen et al. 1997, $1999)$, and also the location of very high-density $\left(n>10^{3} \mathrm{~cm}^{-3}\right)$ shocked gas (Arikawa et al. 1999; Reach et al. 2005). The shelllike radio emission (Long et al. 1991; Dubner et al. 2000) peaks at the northern and northeastern boundaries where interaction with the molecular cloud is established. Further indication of the influence of W 28 on its surroundings is the expanding HI void at a distance $\sim 1.9 \mathrm{kpc}$ (Velázquez et al. 2002). The X-ray emission, which overall is well-explained by a thermal model, peaks in the SNR centre but has local enhancements in a region overlapping the northeastern SNR/molecular cloud interaction (Rho \& Borkowski 2002).
In the neighbourhood of W 28 are the radio-bright HII regions M 20 (Trifid Nebula at $d \sim 1.7 \mathrm{kpc}$ Lynds et al. 1985 - with open cluster NGC 6514), M 8 (Lagoon Nebula at $d \sim 2 \mathrm{kpc}$ Tothill et al. 2002 - containing the open clusters NGC 6523 and NGC 6530) and the ultra-compact HII region W 28A2, all of which are representative of the massive star formation taking place in the region. Further discussion concerning the active star formation in this region may be found in van den Ancker et al. (1997) and references therein. Additional SNRs in the vicinity of W 28 have also been identified: G6.67-0.42 and G7.06-0.12 (Yusef-Zadeh et al. 2000), G5.55+0.32, G6.10+0.53 and G7.20+0.20 (Brogan et al. 2006). The pulsar PSR J1801-23 spin-down luminosity $\dot{E} \sim 6.2 \times$ $10^{34} \mathrm{erg} \mathrm{s}^{-1}$ and distance $d=13.5 \mathrm{kpc}$ (based on its dispersion measure) is at the northern radio edge (Kaspi 1993). More recent discussion (Claussen et al. 2002) assigns a lower limit of $9.4 \pm 2.4 \mathrm{kpc}$ for the pulsar distance.

W 28 has also been linked to $\gamma$-ray emission detected at $E>300 \mathrm{MeV}$ by COS-B (Pollock 1985) and $E>100 \mathrm{MeV}$ by EGRET (Sturner \& Dermer 1995; Esposito et al. 1996; Zhang et al. 1998). The EGRET source, listed in the 3rd catalogue (Hartman et al. 1999) as 3EG J1800-2338, is positioned at the southern edge of the radio shell. We have also performed an analysis of EGRET data, with additional data not included in the 3rd catalogue, and results are discussed later in this paper.

Previous observations of the W 28 region at VHE energies by the CANGAROO-I telescope revealed no evidence for such emission (Rowell et al. 2000) and upper limits at the $\sim 0.2$ to 0.5 Crab-flux level for energies $E>1.5 \mathrm{TeV}(1.1$ to $2.9 \times$ $10^{-11} \mathrm{erg} \mathrm{cm}^{-2} \mathrm{~s}^{-1}$ ) were set for various regions.

\section{Results at VHE and $E>100 \mathrm{MeV} \gamma$-ray energies}

\subsection{HESS VHE analysis and results}

The High Energy Stereoscopic System (HESS) was used to observe the W 28 region. Operating in the Southern Hemisphere, HESS consists of four identical 13 m diameter Cherenkov telescopes (Bernlohr et al. 2003). HESS employs the stereoscopic imaging atmospheric Cherenkov technique, and is sensitive to $\gamma$-rays above an energy threshold of $\sim 0.1 \mathrm{TeV}$ (Funk et al. 2004). An angular resolution of $5^{\prime}$ to $6^{\prime}$ (Gaussian standard deviation) on an event-by-event basis is achieved, and the large field of view $(\mathrm{FoV})$ with full width at half maximum $F W H M \sim 3.5^{\circ}$ permits survey coverage in a single pointing. A point source sensitivity approaching $0.01 \mathrm{Crab}$ flux $\left(\sim 10^{-13} \mathrm{erg} \mathrm{cm}^{-2} \mathrm{~s}^{-1}\right.$ at $\left.1 \mathrm{TeV}\right)$ is achieved for a $5 \sigma$ detection after $\sim 25 \mathrm{~h}$ observation. Further details concerning HESS can be found in Hinton (2004) and references therein.

The total observation time covering the $\mathrm{W} 28$ region amounts to $\sim 42 \mathrm{~h}$ in a series of runs (with typical duration $\sim 28 \mathrm{~min}$ ) spread over the 2004, 2005 and 2006 seasons. Runs were accepted for analysis if they met quality control criteria based on the recorded rate of isotropic CR background events, the number of malfunctioning pixels in each camera, the calibration and the tracking performance (see Aharonian et al. 2004b for details).

Data were analysed using the moment-based Hillas analysis procedure, the same used in the analysis of the inner Galactic Plane Scan datasets (Aharonian et al. 2005a, 2006a). Observations covered a range of zenith angles leading to energy thresholds of $\sim 320 \mathrm{GeV}$ with hard cuts (Cherenkov image integrated intensity or size $>200$ photoelectrons) and $\sim 150 \mathrm{GeV}$ for standard cuts (size $>80$ photoelectrons). Hard cuts were used in VHE $\gamma$-ray images, source location studies and energy spectra. 


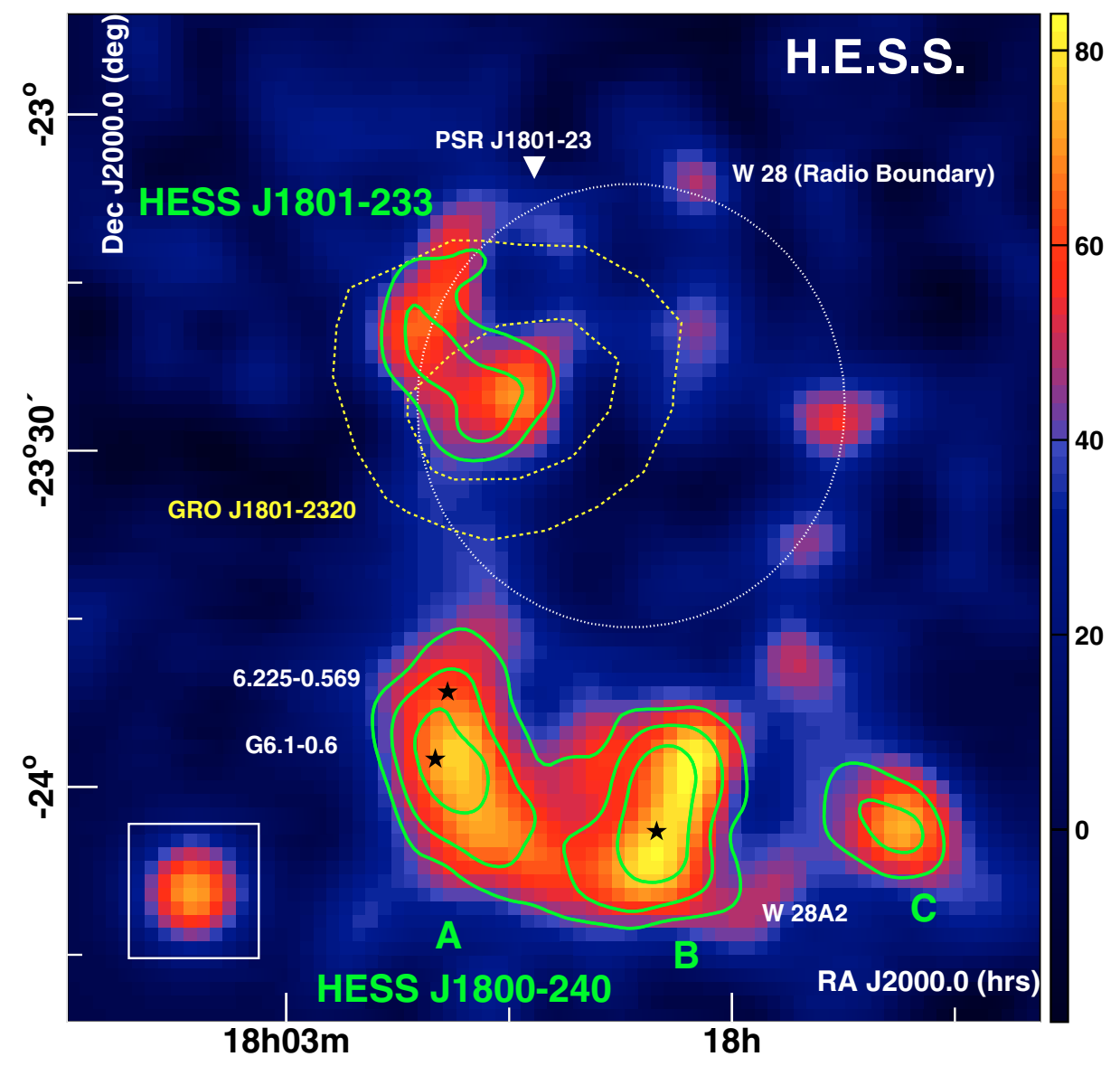

Fig. 1. Image $\left(1.5^{\circ} \times 1.5^{\circ}\right)$ of the VHE $\gamma$-ray excess counts (events), corrected for exposure and smoothed with a Gaussian of radius $4.2^{\prime}$ (standard deviation). Overlaid are solid green contours of VHE excess (pre-trial) significance levels of 4, 5, and $6 \sigma$, after integrating events within an oversampling radius $\theta=0.1^{\circ}$ appropriate for pointlike sources. The thin-dashed circle depicts the approximate radio boundary of the SNR W 28 guided predominantly by the bright northern emission (see Dubner et al. 2000; Brogan et al. 2006). Identified here are VHE source regions HESS J1801-233 to the northeast, and a complex of sources HESS J1800-240 (A, B $\& \mathrm{C})$ to the south of W 28. Also indicated are: HII regions (black stars); W 28A2 (see text), G6.1-0.6 (Kuchar \& Clark 1997), 6.225-0.569 (Lockman 1989); The 68\% and $95 \%$ location contours (thick-dashed yellow lines) of the $E>100 \mathrm{MeV}$ EGRET source GRO J1801-2320; the pulsar PSR J1801-23 (white triangle). The inset to the bottom left depicts a pointlike source for this analysis after the Gaussian smoothing applied to the main image.
In addition, Standard cuts were used in energy spectra in order to increase the energy coverage of extracted spectra. Generally consistent results were obtained using an alternative analysis based on a model of Cherenkov image parameters (de Naurois 2006), which also utilises an independent calibration and lower cut on image size of $>60$ photoelectrons. A forthcoming paper will highlight results in detail from this analysis, which achieves improved sensitivities at lower thresholds compared to the pure Hillas-based analysis.

The VHE $\gamma$-ray image (Fig. 1) reveals two sites of VHE $\gamma$-ray emission in the direction of the northeastern and southern boundaries of the W 28 SNR. The colour scale in this figure depicts the Gaussian-smoothed VHE excess counts above a CR background estimate according to the template model (Rowell 2003), along with significance contours obtained after integrating events within a radius of $0.1^{\circ}$ from each bin centre (appropriate for pointlike source searching). Similar images were obtained using alternative CR background models. A smoothing radius of $4.2^{\prime}$ was used to sufficiently smooth out random fluctuations in the image. An assessment of the VHE post-trial significances was made from our original search for marginally extended sources, which employed an a priori integration radius $\theta=0.2^{\circ}$. Under this scheme we applied $\sim 2.2 \times 10^{5}$ trials (a very conservative value applied to these data) accumulated in searching for sources in the inner Galactic Plane (as in Aharonian 2005a). The pre-trial significance of the VHE sources, at $\geq+7 \sigma$, is therefore converted to a post-trial significance of $\geq+5 \sigma$.

Based on the significance contours in Fig. 1, we assign labels to the northeastern source, HESS J1801-233, and to the complex of sources to the south, HESS J1800-240, according to their best fit positions (fitting a 2D Gaussian and ellipse respectively to the unsmoothed excess map). Three components of HESS J1800-240 are identified, labeled here A, $\mathrm{B}$ and $\mathrm{C}$ from East to West. These components represent local peaks $\sim 2 \sigma$ above their surrounds. Although not convincingly resolved under this analysis these components may comprise separate sources (or at least in part) due to their possible relationship with distinct multiwavelength counterparts (discussed later).

Differential photon energy spectra were extracted from HESS J1801-233 and all three components of HESS J1800-240. Spectra were well-fit by pure power laws $\left(\mathrm{d} N / \mathrm{d} E=k(E / 1 \mathrm{TeV})^{-\Gamma}\right)$ with photon indices $\Gamma \sim 2.5$ to 2.7 in the energy range $\sim 0.3$ to $\sim 5 \mathrm{TeV}$ (see Table 1 for results). Spectral fits were obtained using fluxes from a combination of hard and standard cuts to maximise the energy coverage. Spectral analysis employed the reflected background model (Berge et al. 2007), in which control regions reflected through each tracking position (taking care to avoid known VHE $\gamma$-ray sources) were used to estimate the CR background. Within the statistical and systematic errors, the photon indices appear consistent throughout HESS J1800-240. Except for HESS J1800-240C, all of the VHE sources appear extended with intrinsic radii of $\sim 10^{\prime}$. At a distance of $2 \mathrm{kpc}$, the VHE source luminosities in the energy range 0.3 to $3 \mathrm{TeV}$ would be on the order of $10^{33} \mathrm{erg} \mathrm{s}^{-1}$.

\subsection{EGRET E $>100 \mathrm{MeV}$ analysis and results}

We have also analysed EGRET data for the W 28 region, using CGRO observation cycles (OC) 1 to 6 . This slightly expands on the dataset of the 3rd EGRET catalogue (using OCs 1 to 4; Hartman et al. 1999), which revealed the pointlike source, 3EG J1800-2338 $(E>100 \mathrm{MeV})$. Our analysis confirms 
Table 1. Numerical summary ${ }^{\dagger} \ddagger$ for the VHE and $E>100 \mathrm{MeV}$ sources in the W 28 region including positional and spectral information.

\begin{tabular}{|c|c|c|c|c|c|c|c|}
\hline \multirow[b]{2}{*}{ Name } & \multicolumn{2}{|c|}{ Best fit position (J2000.0) } & \multirow[b]{2}{*}{${ }^{1} \sigma_{\mathrm{src}}[\mathrm{deg}]$} & \multirow[b]{2}{*}{${ }^{2} S[\sigma]$ (evts) } & \multicolumn{2}{|c|}{ Spectral analysis } & \multirow[b]{2}{*}{${ }^{5} L$} \\
\hline & RA [deg] & Dec [deg] & & & ${ }^{3} k$ & ${ }^{4} \Gamma$ & \\
\hline HESS J1801-233 & $270.426 \pm 0.031$ & $-23.335 \pm 0.032$ & $0.17 \pm 0.03$ & $+7.9(281)$ & $7.50 \pm 1.11 \pm 0.30$ & $2.66 \pm 0.27$ & 1.5 \\
\hline HESS J1800-240A ${ }^{\S}$ & $270.491 \pm 0.001$ & $-23.962 \pm 0.001$ & 0.15 & $+6.0(180)$ & $7.65 \pm 1.01 \pm 0.50$ & $2.55 \pm 0.18$ & 1.5 \\
\hline HESS J1800-240B ${ }^{\S}$ & $270.110 \pm 0.002$ & $-24.039 \pm 0.009$ & 0.15 & $+7.8(236)$ & $7.58 \pm 0.90 \pm 0.15$ & $2.50 \pm 0.17$ & 1.4 \\
\hline HESS J1800-240C & $269.715 \pm 0.014$ & $-24.052 \pm 0.006$ & $0.02 \pm 0.15$ & $+4.5(71)$ & $4.59 \pm 0.89 \pm 0.20$ & $2.31 \pm 0.35$ & 0.8 \\
\hline HESS J1800-240 ${ }^{\S \S}$ & $270.156 \pm 0.044$ & $-23.996 \pm 0.022$ & $\begin{array}{l}0.32^{\mathrm{RA}} \pm 0.05 \\
0.17^{\mathrm{Dec}} \pm 0.03\end{array}$ & $+10.3(652)$ & $18.63 \pm 1.85 \pm 1.20$ & $2.49 \pm 0.14$ & 3.6 \\
\hline GRO J1801-2320 & $270.360 \pm 0.150$ & $-23.340 \pm 0.150$ & - & +13.2 & $3.35 \pm 0.52$ & $2.16 \pm 0.10$ & 480.0 \\
\hline
\end{tabular}

$\dagger$ VHE photon spectra are derived from a region of radius $\theta=\sqrt{0.1^{2}+\sigma_{\text {src }}^{2}}$ centered on each source's position unless otherwise indicated. † In spectra, a function $\mathrm{d} N / \mathrm{d} E=k E^{-\Gamma} \mathrm{ph} \mathrm{cm}^{-2} \mathrm{~s}^{-1} \mathrm{TeV}^{-1}$ is fitted. $E$ is in TeV units (HESS data); GeV units (EGRET data).

1. Fitted intrinsic source size (Gaussian std. dev.).

2. Statistical significance and excess events in brackets; for HESS sources using Li \& Ma (1983); for EGRET sources given by $S=\sqrt{T_{\mathrm{s}}}$ for

$T_{\mathrm{s}}$ defined by Mattox et al. (1996).

3. For HESS sources: $\times 10^{-13} \mathrm{ph} \mathrm{cm}^{-2} \mathrm{~s}^{-1} \mathrm{TeV}^{-1}$ at $1 \mathrm{TeV}$ (with statistical and systematic errors); For EGRET sources: $\times 10^{-2} \mathrm{ph}^{-2} \mathrm{~s}^{-1} \mathrm{GeV}^{-1}$ at $1 \mathrm{GeV}$ (with statistical errors).

4. Only statistical errors indicated. Systematic error is estimated at \pm 0.2 .

5. Luminosity $\times 10^{33} \mathrm{erg} \mathrm{s}^{-1}$ at $2 \mathrm{kpc}(0.3$ to $3 \mathrm{TeV}$ for HESS; 0.04 to $6 \mathrm{GeV}$ for EGRET).

$\S$ Due to cross contamination between components A \& B, a fixed value of $\sigma_{\text {src }}=0.15^{\circ}$ estimated visually from Fig. 1 was used.

$\S \S$ Spectrum extracted from a $0.8^{\circ} \times 0.6^{\circ}$ elliptical region encompassing all components $\mathrm{A}, \mathrm{B}, \mathrm{C}$, and matching the size of the corresponding molecular cloud.

the presence of a pointlike $E>100 \mathrm{MeV}$ source in this region, here labeled GRO J1801-2320 (for $E>100 \mathrm{MeV}$ ). GRO J1801-2320 appears slightly shifted $\left(\sim 0.2^{\circ}\right)$ with respect to the $3 \mathrm{EG}$ position. The $3 \mathrm{EG}$ position refers to a $E>100 \mathrm{MeV}$ determination based on the diffuse model as of Hunter et al. (1997). Our dedicated analysis of archival EGRET data comprises different analysis compared to the 3EG catalogue. We first employed the finalised EGRET instrumental responses, which were made available by 2001 and are considered mandatory for investigating an EGRET source under conditions applicable from the end of OC 4 (narrow field of view modus; rapidly deteriorating spark chamber efficiency; and other issues). Second, we restricted the analysis both in narrowing the data selection to pointing angles with respect to our region of interest, which avoids the need to invoke a wide-angle point spread function (PSF). Thirdly, the imprecision of the interstellar emission model was countered via adjustments on analysis parameters gmult and gbias to account for local deviations from the large-scale diffuse emission model in the region of interest. The $68 \%$ and $95 \%$ location contours of GRO J1801-2320 are plotted in Fig. 1, and match well the location of HESS J1801-233. Since however the EGRET degree-scale PSF easily encompasses both of the VHE sources, we cannot rule out a relationship with HESS J1800-240. For the energy spectrum of GRO J1801-2320, we have used the flux points extracted at the position of 3EG J1800-2338 as negligible differences were found between ours and that obtained at the nominal $3 \mathrm{EG}$ position. Fitting a pure power law we obtained a spectral index of $\Gamma=2.16 \pm 0.10$, quite consistent with the published value from Hartman et al. (1999). Comparisons are made with the VHE spectrum of HESS J1801-233 and HESS J1800-240 in Sect. 5.

\section{NANTEN and other observations of molecular clouds}

In searching for molecular cloud counterparts to the VHE sources, we analysed ${ }^{12} \mathrm{CO}(J=1-0)$ molecular line observations taken by the $4-\mathrm{m} \mathrm{mm} / \mathrm{sub}-\mathrm{mm}$ NANTEN telescope, at Las Campanas Observatory, Chile (Mizuno \& Fukui 2004). The
NANTEN Galactic Plane Survey data of 1999 to 2003 (see Matsunaga et al. (2001) and references therein for details) were used, and for the W 28 region, the survey grid spacing was 4'.

Figure 2 (upper left panel) shows the ${ }^{12} \mathrm{CO}(J=1-0)$ image integrated over the Local Standard of Rest velocity $\left(V_{\mathrm{LSR}}\right)$ range 0 to $10 \mathrm{~km} \mathrm{~s}^{-1}$, while the right panel shows the image integrated over the range $V_{\mathrm{LSR}}=10$ to $20 \mathrm{~km} \mathrm{~s}^{-1}$. Two prominent ${ }^{12} \mathrm{CO}$ features representing molecular clouds centred at $(l, b)=\left(6.7^{\circ}\right.$, $\left.-0.3^{\circ}\right)$ and $(l, b)=\left(5.9^{\circ},-0.4^{\circ}\right)$ spatially correspond with the VHE $\gamma$-ray emission. As shown in Fig. 2, these molecular clouds span both $V_{\mathrm{LSR}}$ ranges. According to the Galactic rotation model of Brand \& Blitz (1993), these $V_{\mathrm{LSR}}$ ranges formally correspond to kinematic distances of approximately 0 to $\sim 2.5 \mathrm{kpc}$ (overlapping the Sagittarius arm), and 2.5 to $\sim 4 \mathrm{kpc}$ (reaching the Scutum-Crux arm) respectively. Given the uncertainties in rotation models close to the Galactic centre, such $V_{\mathrm{LSR}}$ ranges would cover the distance estimates for W 28, the most prominent SNR in the region. Much discussion has centred on the systemic velocity (SV) of W 28 (and hence its distance), and how much W 28 has influenced matter in the region. $\mathrm{H} \alpha$ (Radhakrishman et al. 1972) and HI absorption features (Lozinskaya et al. 1981) have suggested $\mathrm{SV} \sim 18 \mathrm{~km} \mathrm{~s}^{-1}$. Claussen et al. (1997) have pointed to $\mathrm{SV} \sim 17 \mathrm{~km} \mathrm{~s}^{-1}$. More recent HI studies by Velázquez et al. (2002) suggest $\mathrm{SV}=+7 \mathrm{~km} \mathrm{~s}^{-1}$ (which leads to the distance estimate for $\mathrm{W} 28$ at $\sim 1.9 \mathrm{kpc}$ ). They also suggest a HI shell may also extend over the $V_{\mathrm{LSR}}=-25$ to $+38 \mathrm{~km} \mathrm{~s}^{-1}$ range, giving rise to a shock speed of $\sim 30 \mathrm{~km} \mathrm{~s}^{-1}$. Torres et al. (2003) and Reach et al. (2005) have also studied the large-scale ${ }^{12} \mathrm{CO}(J=1-0)$ emission for this region using the survey data of Dame et al. (2001), suggesting that the parent molecular cloud under the influence of W 28 is presently centred at $V_{\mathrm{LSR}} \sim 19 \mathrm{~km} \mathrm{~s}^{-1}$. The Galactic longitude-velocity (l-v) diagram (bottom panels of Fig. 2) from our NANTEN data integrated over the Galactic latitude ranges $b=-0.125^{\circ}$ to $-0.5^{\circ}$ and $b=-0.125^{\circ}$ to $-0.7^{\circ}$ shows the distribution of molecular material in relation to the SV of W 28 from the HI studies of Velázquez. The wider, latter $b$ range shows the effect of including the cloud component overlapping HESS J1800-240A. A void or dip in CO emission appears at a similar $V_{\mathrm{LSR}}$ range as found in the HI data, with 

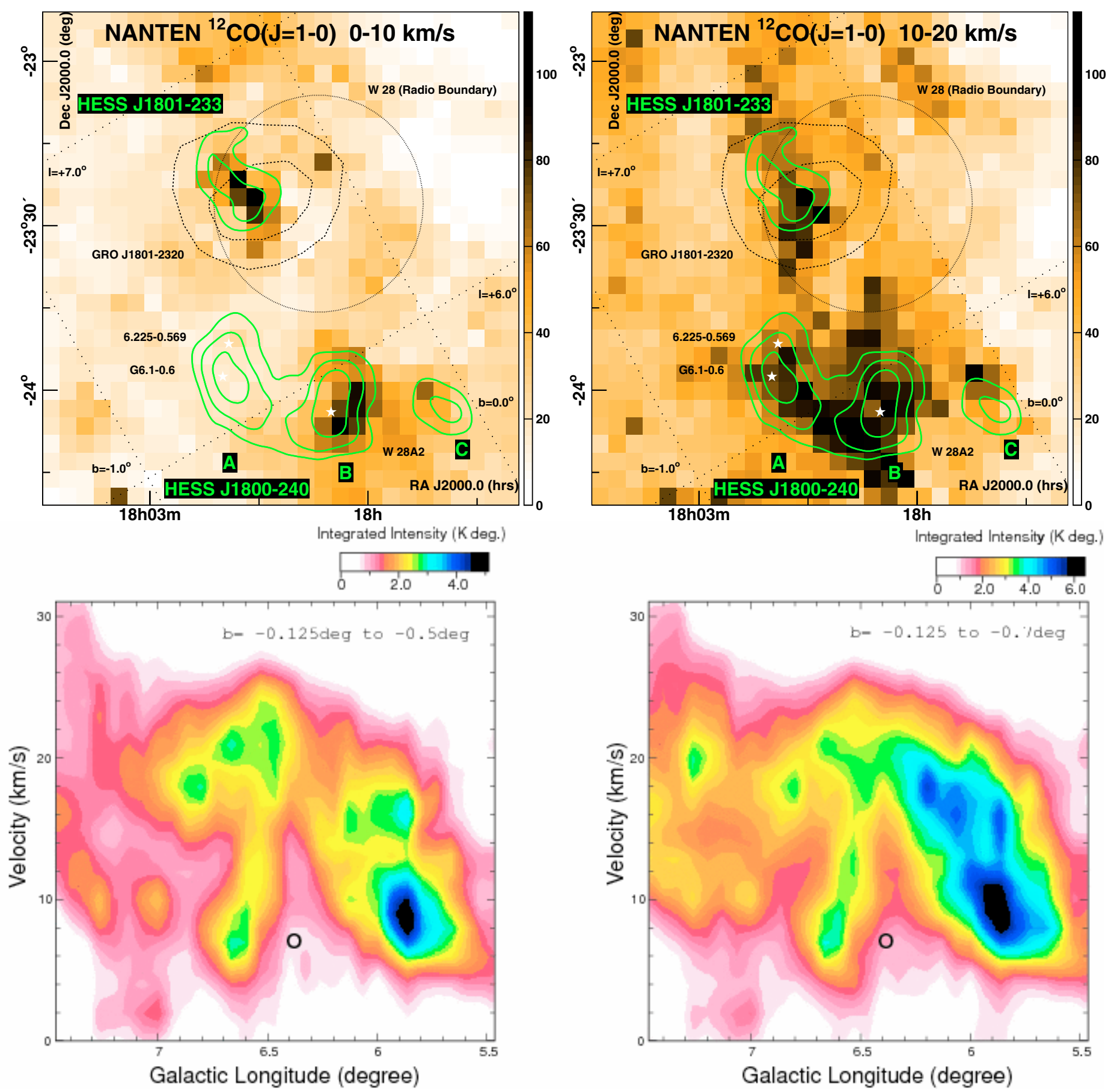

Fig. 2. Upper Left: NANTEN ${ }^{12} \mathrm{CO}(J=1-0)$ image of the $\mathrm{W} 28$ region (linear scale in $\mathrm{K} \mathrm{km} \mathrm{s}^{-1}$ ) for $V_{\mathrm{LSR}}=0$ to $10 \mathrm{~km} \mathrm{~s}{ }^{-1}$ with $\mathrm{VHE}$ $\gamma$-ray significance contours overlaid (green) - levels 4, 5, $6 \sigma$ as in Fig. 1. The radio boundary of W 28, The 68\% and 95\% location contours of GRO J1801-2320 and the location of the HII region W 28A2 (white stars) are indicated. Upper Right: $\mathrm{NANTEN}{ }^{12} \mathrm{CO}(J=1-0)$ image for $V_{\mathrm{LSR}}=10$ to $20 \mathrm{~km} \mathrm{~s}^{-1}$ (linear scale and same maxima as for upper left panel). Bottom panels: distribution of CO emission over the Galactic longitude and $V_{\mathrm{LSR}}$ plane integrated over Galactic latitude $b$ ranges $-0.125^{\circ}$ to $-0.5^{\circ}$ (left) and $-0.125^{\circ}$ to $-0.7^{\circ}$ (right). The latter range is used to show the effect of extending the latitude range to encompass component A of HESS J1800-240. The bold circle indicates the suggested systemic velocity $\left(7 \mathrm{~km} \mathrm{~s}^{-1}\right)$ of W 28 from the HI studies of Velázquez et al. (2002).

much of the molecular material appearing to surround the void in positive $V_{\mathrm{LSR}}$ values with respect to the $\mathrm{SV}$ of $\mathrm{W} 28$. A similar longitude-velocity picture was revealed by Torres et al. (2003) (see their Fig. 22).

The $V_{\mathrm{LSR}}=0$ to $10 \mathrm{~km} \mathrm{~s}^{-1}$ component of the northeast cloud overlapping HESS J1801-233 is already well studied (see Reach et al. 2005 and references therein). Shocked ${ }^{12} \mathrm{CO}(J=3-2)$ molecular gas as indicated by a broad wing-like line dispersion
(Arikawa et al. 1999 - hereafter A99; using the James Clerk Maxwell Telescope (JCMT); in 15" grid steps) and a high concentration of OH masers (Claussen et al. 1997), suggests material here has been compressed by the SNR shock in W 28. The line dispersion, $\Delta V \leq 70 \mathrm{~km} \mathrm{~s}^{-1}$, is an indicator of the SNR shock speed in this particular region. The unshocked gas was also mapped by $\mathrm{A} 99$ via ${ }^{12} \mathrm{CO}(J=1-0)$ observations with the Nobeyama $45 \mathrm{~m}$ telescope (in $34^{\prime \prime}$ grid steps for $V_{\mathrm{LSR}}=+4$ 

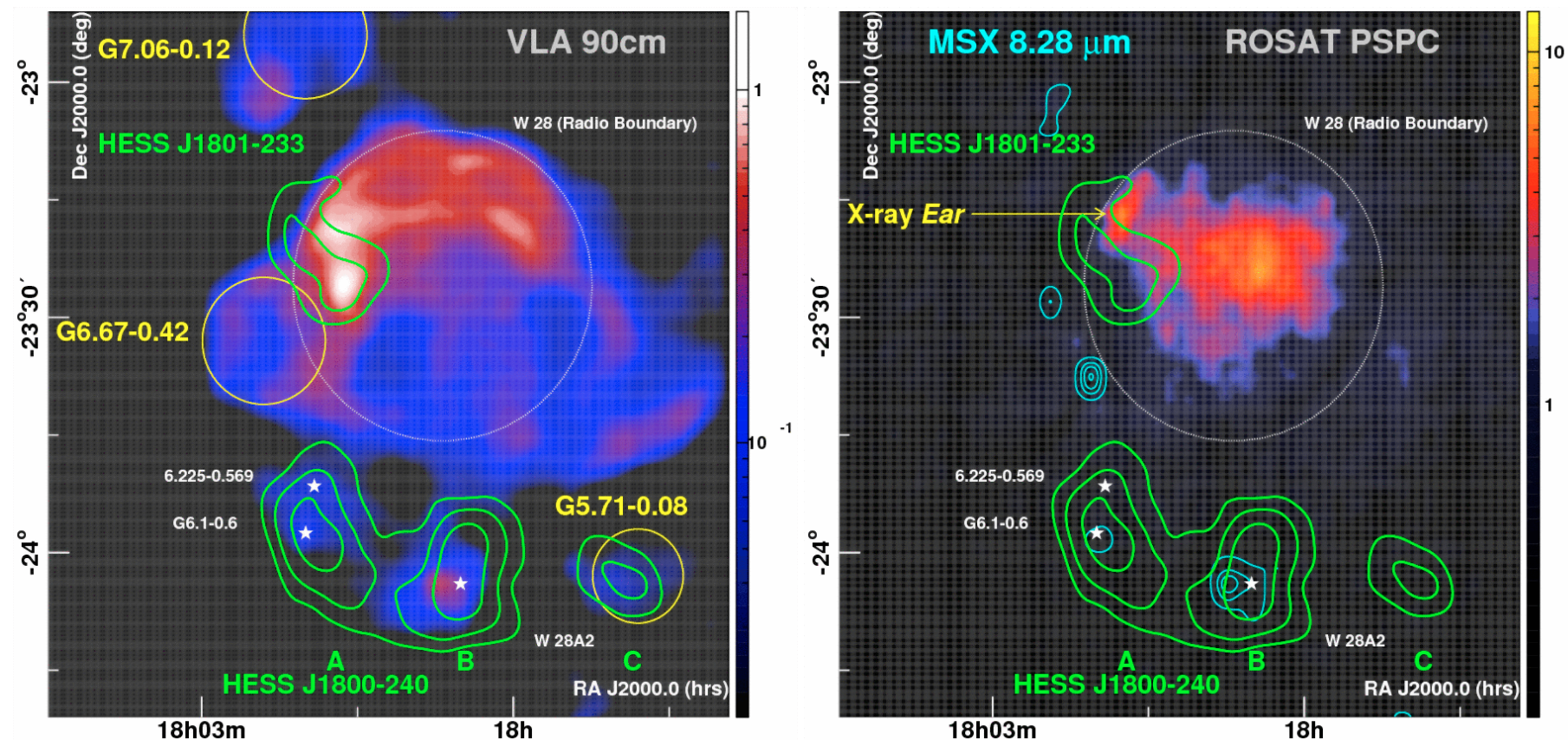

Fig. 3. Left: VLA $90 \mathrm{~cm}$ radio image from Brogan et al. (2006) in $\mathrm{Jy} \mathrm{beam}^{-1}$ (rebinned by a factor 1.2 compared to the original). The VHE significance contours (green) from Fig. 1 are overlaid along with the HII regions (white stars) and the additional SNRs and SNR candidates (with yellow circles indicating their location and approximate dimensions) discussed in the text. Right: ROSAT PSPC image -0.5 to $2.4 \mathrm{keV}$ (smoothed counts per bin from Rho \& Borkowski et al. 2002). Overlaid are contours (cyan - 10 linear levels up to $5 \times 10^{-4} \mathrm{~W} \mathrm{~m}^{-2} \mathrm{sr}^{-1}$ ) from the MSX $8.28 \mu \mathrm{m}$ image. Other contours and objects are as for the left panel. The X-ray Ear representing a peak at the northeastern edge is indicated.

to $+9 \mathrm{~km} \mathrm{~s}^{-1}$ ). The shocked and unshocked gas extends to the northeast and northern boundaries of W 28 (see Fig. 3 of A99), and it appears just their northeastern components are positionally coincident with the VHE emission of HESS J1801-233. A99 estimate the mass and average density of the shocked gas at $M \sim 2 \times 10^{3} M_{\odot}$ and $n \sim 10^{4} \mathrm{~cm}^{-3}$ respectively. For the unshocked gas, A99 obtained $M \sim 4 \times 10^{3} M_{\odot}$ and $n \sim 10^{3} \mathrm{~cm}^{-3}$ respectively. The $V_{\mathrm{LSR}}=10$ to $20 \mathrm{~km} \mathrm{~s}^{-1}$ range in our NANTEN data also reveals additional molecular clouds along the line of sight that could contribute to the VHE emission.

The southern cloud overlaps all components of HESS J1800-240, with a dominant fraction of the cloud overlapping components A and B. The component of this cloud visible in the $V_{\mathrm{LSR}}=0$ to $10 \mathrm{~km} \mathrm{~s}^{-1}$ range coincides well with HESS J1800-240B and the HII region W 28A2. The strongest $\mathrm{CO}$ temperature peak of this component at $(l, b)=\left(5.9^{\circ},-0.4^{\circ}\right)$ is within $0.02^{\circ}$ of $\mathrm{W} 28 \mathrm{~A} 2$, and is likely the dense material surrounding this HII region. Moreover the peak's velocity at $V_{\mathrm{LSR}} 9-10 \mathrm{~km} \mathrm{~s}^{-1}$ (with dispersion of $\sim 15 \mathrm{~km} \mathrm{~s}^{-1}$ ), suggests a distance $(\sim 2.4 \mathrm{kpc})$ similar to that of W 28A2 ( 2 kpc; Acord et al. 1997), and also W 28. In the $V_{\mathrm{LSR}}=10 \mathrm{~km} \mathrm{~s}^{-1}$ to $20 \mathrm{~km} \mathrm{~s}^{-1}$ range, molecular material appears to coincide with all three VHE components of HESS J1800-240. In particular, HESS J1800-240A and C have molecular cloud overlaps only in this latter $V_{\mathrm{LSR}}$ range.

Using the relation between the hydrogen column density $N\left(\mathrm{H}_{2}\right)$ and the ${ }^{12} \mathrm{CO}(J=1-0)$ intensity (the X-factor) $W\left({ }^{12} \mathrm{CO}\right)$, $N\left(\mathrm{H}_{2}\right)=1.5 \times 10^{20}\left[W\left({ }^{12} \mathrm{CO}\right) /\left(\mathrm{K} \mathrm{km} \mathrm{s}^{-1}\right)\right]\left(\mathrm{cm}^{-2}\right)$ (Strong et al. 2004), we estimate a total mass for the northeastern cloud from our NANTEN data at $\sim 5 \times 10^{4} M_{\odot}$ for $d=2 \mathrm{kpc}$ within an elliptical region of diameter $0.2^{\circ} \times 0.4^{\circ}(7 \times 14 \mathrm{pc}$; centred on HESS J1801-233) for the velocity range $0-25 \mathrm{~km} \mathrm{~s}^{-1}$. An average density (for neutral hydrogen) of $\sim 1.4 \times 10^{3} \mathrm{~cm}^{-3}$ is also derived. Similarly the total mass of the southern cloud is estimated at $\sim 1.0 \times 10^{5} M_{\odot}$ for $d=2 \mathrm{kpc}$ and combining clouds from a circular area of radius $0.15^{\circ}(5 \mathrm{pc})$ for the velocity range $12-20 \mathrm{~km} \mathrm{~s}^{-1}$, and area $0.3^{\circ} \times 0.6^{\circ}(10.5 \times 21 \mathrm{pc})$ in diameter for the velocity range $0-12 \mathrm{~km} \mathrm{~s}^{-1}$ (both regions are centred on HESS J1800-240B). The corresponding average density is $\sim 1.0 \times 10^{3} \mathrm{~cm}^{-3}$. By integrating over the rather broad $0-20 \mathrm{~km} \mathrm{~s}^{-1}$ and $0-25 \mathrm{~km} \mathrm{~s}^{-1}$ ranges we assume that the molecular material along this line of sight is physically connected at the same distance (for example $d \sim 2 \mathrm{kpc}$ ) and possibly distrupted or shocked by a local energy source. Systematic effects in the mass estimates arise from the velocity crowding in this part of the Galactic plane, and also the broad velocity range for which X-factor used above may not necessary apply. In the latter case, the X-factor may underestimate the cloud mass since an appreciable fraction of gas may be heated under the assumption of distrupted and/or shock-heated gas. One must allow for $\sim 4 \mathrm{kpc}$ distances for some or even all of the $V_{\mathrm{LSR}}>10 \mathrm{~km} \mathrm{~s}^{-1}$ cloud components, and therefore the conclusion that they are not related to $\mathrm{W} 28$ and other interesting objects at $d \sim 2 \mathrm{kpc}$. If the clouds are related, W 28 could play a disrupting role. The level of this disruption is however unclear since several other plausible candidates related to the star formation (discussed later) in this region could also contribute. Some other molecular cloud complexes have also been discussed as possibly disrupted by adjacent SNRs and/or energetic sources (e.g. Yamaguchi et al. 1999; Moriguchi et al. 2000). In Table 2, we present a full summary of cloud masses and densities (for regions centered on the VHE source coordinates as in Table 1) for various combiniations of cloud components and distances of 2 and $4 \mathrm{kpc}$. Velocity separation of cloud components are based on their apparent distribution in Fig. 2 (bottom panels).

\section{Radio to X-ray views}

Figure 3 compares the radio (left panel), infrared and X-ray views (right panel) of the $\mathrm{W} 28$ region with the VHE significance 
contours. The Very Large Array (VLA) $90 \mathrm{~cm}$ continuum radio image from Brogan et al. (2006) illustrates the shell-like SNR morphology peaking strongly along the northern and eastern boundaries. HESS J1801-233 can be seen to overlap the northeastern shell of the SNR, coinciding with a strong peak in the $90 \mathrm{~cm}$ continuum emission. We note that a thermal component is likely present in this peak, given its spectral index $\alpha \sim-0.2$ (for $S \propto v^{\alpha}$ ) between 90 and $20 \mathrm{~cm}$ (Dubner et al. 2000). Outlines of the SNRs traced by non-thermal radio emission, G6.67-0.42 and G7.06-0.12 (Yusef-Zadeh et al. 2000; Helfand et al. 2006; labelled as G6.51-0.48 and G7.0-0.1 by Brogan et al. 2006) are also indicated. In addition, Brogan et al. notes that the non-thermal radio arc G5.71-0.08, which overlaps well with HESS J1800-240C, could be a partial shell and therefore an SNR candidate. The distances to G6.67-0.42 and G5.71-0.08 are presently unknown. Directly south of W 28, the ultracompact HII region $\mathrm{W} 28 \mathrm{~A} 2$ is a prominent radio source, and is positioned within $0.1^{\circ}$ of the centroid of HESS J1800-240B. The other HII regions G6.1-0.6 (Kuchar \& Clark 1997) and 6.225-0.569 (Lockman 1989) are also associated with radio emission.

The X-ray morphology as shown (Fig. 3 right panel) in the ROSAT PSPC ( 0.5 to $2.4 \mathrm{keV})$ image from Rho \& Borkowski (2002) reveals the central concentration of X-ray emission, which is predominantly thermal in nature with characteristic temperatures in the range $k T \sim 0.4$ to $2 \mathrm{keV}$. An X-ray peak or Ear lies at the northeastern boundary and just outside the $4 \sigma$ significance contour of HESS J1801-233. A non-thermal component to the ear emission $\left(3 \times 1.5^{\prime}\right)\left(2.1 \times 10^{-14} \mathrm{erg} \mathrm{cm}^{-2} \mathrm{~s}^{-1}\right.$ at $1 \mathrm{keV}$ ) with a power-law index $\Gamma=1.3$ has been suggested by Ueno et al. (2003a) based on XMM-Newton observations in the 0.5 to $10 \mathrm{keV}$ energy range. The total kinetic energy of the SNR is estimated at $\sim 4 \times 10^{50} \mathrm{erg}$, which could be a lower limit due to the possible break-out of the SNR along the southern edge away from the molecular cloud to the north and east (Rho \& Borkowski 2002). The HII regions, W 28A2 and G6.1-0.6 are prominent in the $8.28 \mu \mathrm{m}$ image (Fig. 3 right panel) from the Midcourse Space Experiment (MSX), showing that a high concentration of heated dust still surrounds these very young stellar objects.

\section{Discussion}

Our discovery of VHE $\gamma$-ray emission associated with dense $\left(n \geq 10^{3} \mathrm{~cm}^{-3}\right.$ ) molecular clouds in the W 28 field adds to the list of such associations after the detection of diffuse $\gamma$-ray emission from the Galactic Ridge (Aharonian et al. 2006c), the association of HESS J1834-087 with the old-age SNR W 41 (Lemiere et al. 2005; Albert et al. 2006) and VHE emission discovered from IC 443 (Albert et al. 2007). The VHE/molecular cloud association could indicate a hadronic origin for the parent multi$\mathrm{TeV}$ particles where the $\gamma$-ray emission (multi-GeV to $\mathrm{TeV}$ energies) arises from the decay of neutral pions resulting from the interaction of accelerated protons (and higher $Z$ nuclei) with ambient matter of density $n$. In this case the $\gamma$-ray flux would scale with cloud mass or density, and the total energy in accelerated particles or CRs penetrating the cloud(s). We note that a perfect correlation between the VHE and molecular cloud morphologies is not expected due to complex time and energy-dependent propagation of CR to and within the cloud (see Gabici et al. 2006, for a discussion). Projection effects are also likely to be important for the examples discussed here since the VHE emission could have contributions from clouds at different velocities, not necessarily physically connected to one another. For example the
Table 2. Details for the molecular clouds towards the VHE sources in the W 28 field, assuming a distance $d$.

\begin{tabular}{llllll}
\hline \hline VHE Source & $\begin{array}{l}V_{\mathrm{LSR}} \\
\left(\mathrm{km} \mathrm{s}^{-1}\right)\end{array}$ & $\begin{array}{l}d \\
(\mathrm{kpc})\end{array}$ & ${ }^{\dagger} M$ & ${ }^{\ddagger} n$ & ${ }^{\S} k_{\mathrm{CR}}$ \\
\hline HESS J1801-233 & $0-25$ & 2.0 & 0.5 & 1.4 & 13 \\
HESS J1801-233 & $0-12$ & 2.0 & 0.2 & 2.3 & 32 \\
HESS J1801-233 & $13-25$ & 4.0 & 1.1 & 0.6 & 23 \\
HESS J1800-240 & $0-20$ & 2.0 & 1.0 & 1.0 & 18 \\
HESS J1800-240A & $12-20$ & 4.0 & 1.0 & 0.7 & 28 \\
HESS J1800-240B & $0-12$ & 2.0 & 0.4 & 2.3 & 18 \\
HESS J1800-240B & $12-20$ & 4.0 & 1.5 & 1.2 & 19 \\
\hline
\end{tabular}

${ }^{\dagger}$ Cloud mass $\times 10^{5} M_{\odot} .{ }^{\ddagger}$ Cloud density $\times 10^{3} \mathrm{~cm}^{-3} .{ }^{\S}$ Cosmic-ray density enhancement, $k_{\mathrm{CR}}$ above the local value required to produce the $E>1 \mathrm{TeV}$ VHE $\gamma$-ray emission (using Eq. (10) of Aharonian 1991).

relationship between HESS J1801-233 and the W 28/molecular cloud interaction is not entirely clear due to the overlapping molecular cloud components at $V_{\mathrm{LSR}}>10 \mathrm{~km} \mathrm{~s}^{-1}$.

One should also consider accelerated electrons as the source of $\gamma$-ray emission, via inverse-Compton (IC) scattering of ambient soft photon fields and/or non-thermal Bremsstrahlung from the interaction of electrons with dense ambient matter. Maximum electron energies however may be considerably lower (factor $\sim 10$ or more than that of protons) due to synchrotron cooling in magnetic fields and low shock speeds, in the absence of strong electron replenishment. An assessment of the role of accelerated electrons requires consideration of the nonthermal radio and X-ray emission (where a convincing measurement of the latter is so far lacking), and also magnetic fields in this region. Such observations will also provide constraints on synchrotron emission expected from secondary electrons resulting from primary hadron interactions with ambient matter (as discussed above). Relatively high magnetic fields $B \sim 100\left(n / 10^{4} \mathrm{~cm}^{-3}\right)^{0.5} \mu \mathrm{G}$ are inferred in dense molecular clouds (Crutcher et al. 1999). In addition, higher values are indicated from Zeeman splitting measurements in the compact areas (arcsecond scale) surrounding the $1720 \mathrm{MHz} \mathrm{OH}$ masers of the northeastern interaction region (Hoffman et al. 2005), coinciding with HESS J1801-233. To the north of W 28, another potential source of particle acceleration is PSR J1801-23, where the VHE emission may arise in an asymmetric pulsarwind-nebula (PWN) scenario (a primarily leptonic scenario), similar to HESS J1825-137 (Aharonian et al. 2006d). However with a spin-down power of $\dot{E} \sim 6.2 \times 10^{34} \mathrm{erg} \mathrm{s}^{-1}$ at distance $d>9.4 \mathrm{kpc}$, this pulsar appears unlikely to power any of the $\gamma$-ray sources observed in the region. A PWN scenario would therefore require a so far undetected energetic pulsar.

In the case of a hadronic origin and following Eq. (10) of Aharonian (1991), we can estimate the CR density enhancement factor $k_{\mathrm{CR}}$ in units of the local CR density required to explain the VHE emission, given an estimate for the cloud masses and assumptions on distance. Converting the VHE energy spectra in Table 1 to an integral value for $E>1 \mathrm{TeV}$, assuming distances of 2 and $4 \mathrm{kpc}$ for the various cloud components, and that all the VHE emission in each source is associated with the cloud component under consideration, we arrive at values for $k_{\mathrm{CR}}$ in the range 13 to 32 (Table 2).

Overall, these levels of CR enhancement factor would be expected in the neighbourhood of CR accelerators such as SNRs. If the clouds were all at $\sim 2 \mathrm{kpc}$, an obvious candidate for such particle acceleration is the SNR W 28, the most prominent SNR in the region. Despite its old age, multi-TeV particle acceleration 
may still occur in W 28 (Yamazaki et al. 2006), with protons reaching energies of several 10's of TeV depending on various SNR shock parameters such as speed, size and ambient matter density. In addition, CRs produced at earlier epochs have likely escaped and diffused throughout the region, a situation discussed at length in Aharonian \& Atoyan (1996). Aharonian \& Atoyan show for slow diffusion (diffusion coefficient at $10 \mathrm{GeV}$ $D_{10} \sim 10^{26} \mathrm{~cm}^{2} \mathrm{~s}^{-1}$ as might be expected in dense environments) $\mathrm{CR}$ enhancement factors in the required range could be found in the vicinity (within $30 \mathrm{pc}$ - note that if at $2 \mathrm{kpc}$ distance, HESS J1800-240 would lie $\sim 10$ pc from the southern circular boundary of W 28) of a canonical SNR as an impulsive accelerator up to $\sim 10^{5} \mathrm{yr}$ after the SN explosion (see their Fig. 1). In this sense, W 28 as a source of CRs in the region could be a plausible scenario.

The W 28 field however is a rich star formation region, and several additional/alternative sources of $\mathrm{CR}$ acceleration may be active. The SNR G6.67-0.42 is positioned directly to the southeast of HESS J1801-233 (Fig. 3 left panel) while the SNR G7.06-0.12 is situated $\sim 0.25^{\circ}$ north of HESS J1801-233 and on the west side of the HII region M 20. M 20 itself may also be an energy source for the molecular clouds in this region. The SNR candidate G5.71-0.08 (Brogan et al. 2006) may also be responsible in some way for HESS J1800-24C given the good positional overlap between the two. These radio SNR/SNR candidates are without a distance estimate making it unclear as to how they relate to the molecular clouds in the region. The morphology of HESS J1800-240 displays several peaks, perhaps resulting from changes in cloud density and/or the presence of additional particle accelerators and local conditions. For HESS J1800-24B, a potential energy source is the unusual ultracompact HII region W 28A2 (G5.89-0.39), representing a massive star in a very young phase of evolution. W 28A2 exhibits very energetic bipolar molecular outflows (Harvey \& Forveille 1988; Acord et al. 1997; Sollins et al. 2004) which may arise from the accretion of matter by the progenitor star. The outflow ages are estimated at between $\sim 10^{3}$ to $10^{4}$ yr. Recent observations (Klaassen et al. 2006) suggest both outflows extend over a combined distance of $\sim 2^{\prime}$ ( or $\sim 1.2 \mathrm{pc}$ at $d=2 \mathrm{kpc}$ ), with total kinetic energy of $3.5 \times 10^{46} \mathrm{erg}$. Surrounding the outflows is a very dense $\left(>10^{4} \mathrm{~cm}^{-3}\right)$ molecular envelope of diameter $0.5^{\prime}$ to $1^{\prime}$. Despite the lack of any model to explain multi-TeV particle acceleration in such HII regions, its kinetic energy budget and its spatial overlap with a VHE source makes W 28A2 a tempting candidate for such acceleration. Already, there are two examples of VHE emission possibly related to the environments of hot, young stars - TeV J2032+4130 (Aharonian et al. 2005b) and HESS J1023-575 (Aharonian et al. 2007c). In this context, the HII regions G6.1-0.6 and 6.225-0.569 may also play a similar role in HESS J1800-24A. Among the prominent open clusters in the area, NGC 6523 and NGC $6530 \sim 0.5^{\circ}$ southeast of HESS J1800-240, and NGC 6514 associated with M $20 \sim 0.7^{\circ}$ north of HESS J1801-233 may also provide energy for CR production. Finally, if the VHE emission is associated with truly distant cloud components approaching the Scutum-Crux arm at $\sim 4 \mathrm{kpc}$, undetected background particle accelerators would then play a role.

Figure 4 also compares the EGRET and VHE spectra. Given the degree-scale EGRET PSF, GRO J1801-2320 remains unresolved at scales of the VHE sources. Although the peak of the EGRET emission coincides with HESS J1801-233, we therefore cannot rule out unresolved $\mathrm{MeV} / \mathrm{GeV}$ components from HESS J1800-240. Observations with GLAST will be required to determine the $\mathrm{MeV} / \mathrm{GeV}$ components of the VHE sources.

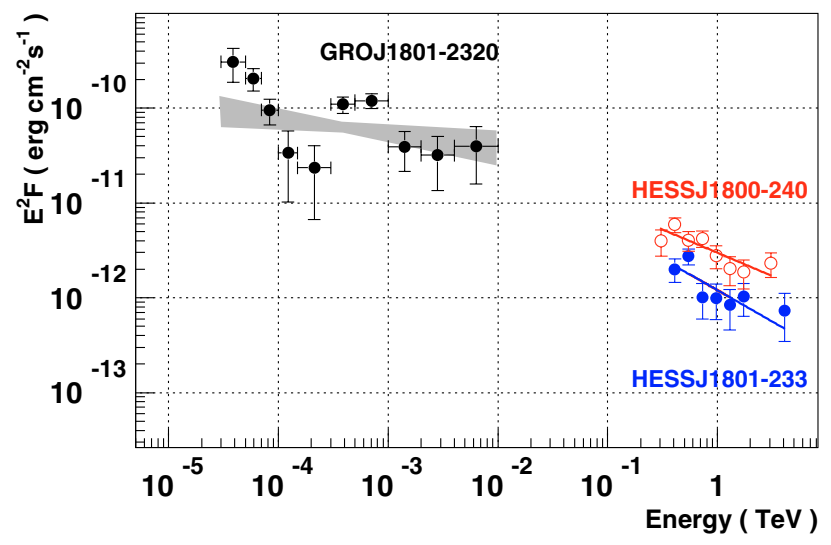

Fig. 4. Energy fluxes of HESS J1801-233 and HESS J1800-240 (for regions defined in Table 1) compared to the $E>100 \mathrm{MeV}$ counterpart GRO J1801-2320. The power law fits and data points (summarised in Table 1) are also indicated: HESS J1801-233 (solid blue line and points); HESS J1800-240 (open red points and solid line); GRO J1801-232 (solid black points and grey $1 \sigma$ confidence band).

\section{Conclusions}

In conclusion, our observations with the HESS $\gamma$-ray telescopes have revealed VHE $\gamma$-ray sources in the field of W 28 which positionally coincide well with molecular clouds. HESS J1801-233 is seen toward the northeast boundary of W 28, while HESS J1800-240 situated just beyond the southern boundary of W 28 comprises three components. Our studies with NANTEN ${ }^{12} \mathrm{CO}(J=1-0)$ data show molecular clouds spanning a broad range in local standard of rest velocity $V_{\mathrm{LSR}}=5$ to $\sim 20 \mathrm{~km} \mathrm{~s}^{-1}$, encompassing the distance estimates for W 28 and various star formation sites in the region. If connected, and at a distance $\sim 2 \mathrm{kpc}$, the clouds may be part of a larger parent cloud possibly disrupted by W 28 and/or additional objects related to the active star formation in the region. Cloud components up to $\sim 4 \mathrm{kpc}$ distance $\left(V_{\mathrm{LSR}}>10 \mathrm{~km} \mathrm{~s}^{-1}\right)$ however, remain a possibility.

The VHE/molecular cloud association could indicate a hadronic origin for the VHE sources in the W 28 field. Under assumptions of connected cloud components at a common distance of $2 \mathrm{kpc}$, or, alternatively, separate cloud components at 2 and $4 \mathrm{kpc}$, a hadronic origin for the VHE emission implies cosmic-ray densities $\sim 10$ to $\sim 30$ times the local value. W 28 could provide such densities in the case of slow diffusion. Additional and/or alternative particle accelerators such as HII regions representing very young stars, other SNRs/SNR candidates and/or several open clusters in the region may also be contributors. Alternatively, if cloud components at $V_{\mathrm{LSR}}>$ $10 \mathrm{~km} \mathrm{~s}^{-1}$ are at distances $d \sim 4 \mathrm{kpc}$, as-yet undetected particle accelerators in the Scutum-Crux arm may be responsible. Detailed modeling (beyond the scope of this paper), and further multiwavelength observations of this region are highly recommended to assess further the relationship between the molecular gas and potential particle accelerators in this complex region, as well as the nature of the acclerated particles. In particular, further sub-mm observations (e.g. at high CO transitions) will provide more accurate cloud mass estimates, and allow to search for disrupted/shocked gas towards the southern VHE sources. Such studies will be valuable in determining whether or not W 28 and other energetic sources have disrupted molecular material at line velocities $>10 \mathrm{~km} \mathrm{~s}^{-1}$. 
Acknowledgements. The support of the Namibian authorities and of the University of Namibia in facilitating the construction and operation of HESS is gratefully acknowledged, as is the support by the German Ministry for Education and Research (BMBF), the Max Planck Society, the French Ministry for Research, the CNRS-IN2P3 and the Astroparticle Interdisciplinary Programme of the CNRS, the UK Particle Physics and Astronomy Research Council (PPARC), the IPNP of the Charles University, the Polish Ministry of Science and Higher Education, the South African Department of Science and Technology and National Research Foundation, and by the University of Namibia. We appreciate the excellent work of the technical support staff in Berlin, Durham, Hamburg, Heidelberg, Palaiseau, Paris, Saclay, and in Namibia in the construction and operation of the equipment. The NANTEN project is financially supported from JSPS (Japan Society for the Promotion of Science) Core-to-Core Program, MEXT Grant-in-Aid for Scientific Research on Priority Areas, and SORST-JST (Solution Oriented Research for Science and Technology: Japan Science and Technology Agency). We also thank Crystal Brogan for the VLA $90 \mathrm{~cm}$ image and the referee for valuable comments.

\section{References}

Acord, J. M., Walmsley, C. M., \& Churchwell, E. 1997, ApJ, 475, 693

Aharonian, F. 1991, Ap\&SS, 180, 305

Aharonian, F., \& Atoyan, A. M. 1996, A\&A, 309, 917

Aharonian, F., Akhperjanian, A. G., Aye, K.-M., et al. (HESS Collab.) 2004a, Nature, 432, 75

Aharonian, F., Akhperjanian, A. G., Aye, K.-M., et al. (HESS Collab.) 2004b, Astropart. Phys., 22, 109

Aharonian, F., Akhperjanian, A. G., Aye, K.-M., et al. (HESS Collab.) 2005a, Science, 307, 1938

Aharonian, F., Akhperjanian, A. G., Beilicke, M., et al. (HEGRA Collab.) 2005b, A\&A, 431, 197

Aharonian, F., Akhperjanian, A. G., Bazer-Bachi A. R., et al. (HESS Collab.) 2005c, A\&A, 437, L7

Aharonian, F., Akhperjanian, A. G., Bazer-Bachi A. R., et al. (HESS Collab.) 2006a, ApJ, 636, 777

Aharonian, F., Akhperjanian, A. G., Bazer-Bachi A. R., et al. (HESS Collab.) 2006b, A\&A, 449, 223

Aharonian, F., Akhperjanian, A. G., Bazer-Bachi A. R., et al. (HESS Collab.) 2006c, Nature, 439, 695

Aharonian, F., Akhperjanian, A. G., Bazer-Bachi A. R., et al. (HESS Collab.) 2006d, A\&A, 460, 365

Aharonian, F., Akhperjanian, A. G., Bazer-Bachi A. R., et al. (HESS Collab.) 2007a, A\&A, 464, 235

Aharonian, F., Akhperjanian, A. G., Bazer-Bachi A. R., et al. 2007b, ApJ, 661, 236

Aharonian, F., Akhperjanian, A. G., Bazer-Bachi, A. R., et al. (HESS Collab.) 2007c, A\&A, 467, 1075

Albert, J., Aliu, E., Anderhub, H., et al. 2006, ApJ, 643, L53

Albert, J., Aliu, E., Anderhub, H., et al. 2007, ApJ, 664, L87

van den Ancker, M. E., Thé, P. S., Feinstein, A., et al. 1997, A\&AS, 123, 63

Arikawa, Y., Tatematsu, K., Sekimoto, Y., \& Takahashi, T. 1999, PASJ, 51, L7

Berge, D., Funk, S., \& Hinton, J. 2007, A\&A, 466, 1219

Berezhko, E. G., \& Völk, H. J. 2006, A\&A, 451, 981

Berezhko, E. G., Pühlhofer, G., \& Völk, H. J. 2007, Proc. 30th ICRC (Merida) [arXiv:0707.4662]

Bernlohr, K., Carrol, O., Cornils, R., et al. 2003, Astropart. Phys., 20, 111

Blandford, R. D., \& Eichler, D. 1987, Phys. Rep., 154, 1

Blondin, J. M., Wright, E. B., Borkowski, K. J., et al. 1998, ApJ, 500, 342

Brand, J., \& Blitz, L. 1993, A\&A, 275, 67

Brogan, C. L., Gelfand, J. D., Gaensler, B. M., et al. 2006, ApJ, 639, L25

Claussen, M. J., Frail, D. A., Goss, W. M., \& Gaume, R. A. 1997, ApJ, 489, 143

Claussen, M. J., Goss, W. M., Frail, D. A., \& Desai, K. 1999, ApJ, 522, 349

Claussen, M. J., Goss, W. M., Desai, K. M., \& Brogan, C. L. 2002, ApJ, 580, 909

Crutcher, R. M. 1999, ApJ, 520, 706

Dame, T. M., Hartman, D., \& Thaddeus, P. 2001, ApJ, 547, 792

Drury, L. O.'C. 1983, Rep. Prog. Phys., 46, 973

Dubner, G. M., Velázquez, P. F., Goss, W. M., \& Holdaway, M. A. 2000, AJ, 120,1933

Esposito, J. A., Hunter, S. D., Kanbach, G., \& Sreekumar, P. 1996, ApJ, 461, 820

Frail, D. A., Goss, W. M., \& Slysh, V. I. 1994, ApJ, 424, L111

Funk, S., Hermann, G., Hinton, J., et al. 2004, Astropart. Phys., 22, 285

Ginzburg, V. L., \& Syrovatskii, S. I. 1964, The Origin of Cosmic Rays (New York: Macmillan)

Gabici, S., Aharonian, F. A., \& Blasi, P. 2006, In Proc. Multi-messenger approach to high energy gamma-rays, Barcelona June 2006, Ap\&SS [arXiv:astro-ph/0610032]
Goudis, C. 1976, Ap\&SS, 40, 91

Hartman, R. C., Bertsch, D. L., Bloom, S. D., et al. 1999, ApJS, 123, 79

Harvey, P. M., \& Forveille, T. 1988, A\&A, 197, L19

Helfand, D. J., Becker, R. H., White, R. L., et al. 2006, AJ, 131, 2525

Hinton, J. A. 2004, New Astron. Rev., 48, 331

Hoffman, I. M., Goss, W. M., Brogan, C. L., \& Claussen, M. J. 2005, ApJ, 620, 257

Hunter, S. D., Bertsch, D. L., Catelli, J. R., et al. 1997, ApJ, 481, 205

Kaspi, V. M., Lyne, A. G., Manchester, R. N., et al. 1993, ApJ, 409, L57

Klaassen, P. D., Plume, R., Ouyed, R., et al. 2006, ApJ, 648, 1079

Koyama, K., Petre, R., Gotthelf, E. V., et al. 1995, Nature, 378, 255

Koyama, K., Kinugasa, K., \& Matsuzaki, K. 1997, PASJ, 49, L7

Kuchar, T. A., \& Clark, F. O. 1997, ApJ, 488, 224

Lemiére, A., et al. (HESS Collab.) 2005, in Proc. 29th ICRC (Pune) 4, 105

Li, T., \& Ma, Y. 1983, ApJ, 272, 317

Lockman, F. J. 1989, ApJS, 71, 469

Long, K. S., Blair, W. P., White, R. L., \& Matsui, Y. 1991, ApJ, 373, 567

Lozinskaya, T. A. 1981, Sov. Astron. Lett. 7, 17

Lynds, B. T., \& O’Neill, E. J., Jr. 1985, ApJ, 294, 578

Matsunaga, K., Mizuno, N., Moriguchi, Y., et al. 2001, PASJ, 53, 1003

Mattox, J. R., Bertsch, D. L., Chiang, J., et al. 1996, ApJ, 461, 396

Mizuno, A., \& Fukui, Y. 2004, ASP Conf. Proc., 317, 59

Moriguchi, Y., Yamaguchi, N., Onishi, T., et al. 2000, PASJ, 53, 1025

de Naurois, M. 2006 [arXiv:astro-ph/0607247]

Pollock, A. M. T. 1985, A\&A, 150, 339

Radhakrishman, V., Goss, W. M., Marray, J. E., et al. 1972, ApJS, 24, 49

Reach, W. T., Rho, J., \& Jarrett, T. H. 2005, ApJ, 618, 297

Rho, J. H., \& Borkowski, K. 2002, ApJ, 575, 201

Rowell, G. P. 2003, A\&A, 410, 389

Rowell, G. P., Naito, T., Dazeley, S. A., et al. 2000, A\&A, 359, 337

Sollins, P. K., Hunter, T. R., Battat, J., et al. 2004, ApJ, 616, L35

Strong, A. W., Moskalenko, I. A. W., Reimer, O., et al. 2004, A\&A, 422, L47

Sturner, S. J., \& Dermer, C. D. 1995, A\&A, 293, L17

Torres, D. F., Romero, G., Dame, T. M., et al. 2003, Phys. Rep., 382, 303

Tothill, N. F. H., White, G. J., Matthews, H. E., et al. 2002, ApJ, 580, 285

Ueno, M., Bamba, A., \& Koyama, K. 2003a, In Proc. 28th ICRC (Tsukuba, Japan), 2401

Velázquez, P. F., Dubner, G. M., Goss, W. M., \& Green, A. J. 2002, AJ, 124, 2145

Wootten, A. 1981, ApJ, 245, 105

Yamaguchi, N., Mizuno, N., Moriguchi, Y., et al. 1999, PASJ, 51, 765

Yamazaki, R., Kazunori, K., Yoshida, T., \& Tsuribo, T. 2006, MNRAS, 371, 1975

Yusef-Zadeh, F., Shure, M., Wardle, M., \& Kassim, N. 2000, ApJ, 540, 842

Zhang, I., \& Cheng, K. S. 1998, A\&A, 335, 234

1 Max-Planck-Institut für Kernphysik, PO Box 103980, 69029 Heidelberg, Germany

e-mail: rowell@physics . adelaide.edu . au

2 Yerevan Physics Institute, 2 Alikhanian Brothers St., 375036 Yerevan, Armenia

3 Centre d'Etude Spatiale des Rayonnements, CNRS/UPS, 9 Av. du Colonel Roche, BP 4346, 31029 Toulouse Cedex 4, France

4 Universität Hamburg, Institut für Experimentalphysik, Luruper Chaussee 149, 22761 Hamburg, Germany

5 Institut für Physik, Humboldt-Universität zu Berlin, Newtonstr. 15, 12489 Berlin, Germany

6 LUTH, UMR 8102 du CNRS, Observatoire de Paris, Section de Meudon, 92195 Meudon Cedex, France

7 DAPNIA/DSM/CEA, CE Saclay, 91191 Gif-sur-Yvette, Cedex, France

8 University of Durham, Department of Physics, South Road, Durham DH1 3LE, UK

9 Unit for Space Physics, North-West University, Potchefstroom 2520, South Africa

10 Laboratoire Leprince-Ringuet, IN2P3/CNRS, École Polytechnique, 91128 Palaiseau, France

11 Laboratoire d'Annecy-le-Vieux de Physique des Particules, IN2P3/CNRS, 9 Chemin de Bellevue, BP 110, 74941 Annecy-leVieux Cedex, France

12 APC, 11 place Marcelin Berthelot, 75231 Paris Cedex 05, France, UMR 7164 (CNRS, Université Paris VII, CEA, Observatoire de Paris). 
13 Dublin Institute for Advanced Studies, 5 Merrion Square, Dublin 2, Ireland

14 Landessternwarte, Universität Heidelberg, Königstuhl, 69117 Heidelberg, Germany

15 Laboratoire de Physique Théorique et Astroparticules, IN2P3/CNRS, Université Montpellier II, CC 70, Place Eugène Bataillon, 34095 Montpellier Cedex 5, France

16 Universität Erlangen-Nürnberg, Physikalisches Institut, ErwinRommel-Str. 1, 91058 Erlangen, Germany

17 Laboratoire d'Astrophysique de Grenoble, INSU/CNRS, Université Joseph Fourier, BP 53, 38041 Grenoble Cedex 9, France

18 Institut für Astronomie und Astrophysik, Universität Tübingen, Sand 1, 72076 Tübingen, Germany

19 LPNHE, Université Pierre et Marie Curie Paris 6, Université Denis Diderot Paris 7, CNRS/IN2P3, 4 place Jussieu, 75252 Paris Cedex 5, France
20 Institute of Particle and Nuclear Physics, Charles University, V Holesovickach 2, 18000 Prague 8, Czech Republic

${ }^{21}$ Institut für Theoretische Physik, Lehrstuhl IV: Weltraum und Astrophysik, Ruhr-Universität Bochum, 44780 Bochum, Germany

22 University of Namibia, Private Bag 13301, Windhoek, Namibia

23 Obserwatorium Astronomiczne, Uniwersytet Jagielloński, Kraków, Poland

24 Nicolaus Copernicus Astronomical Center, Warsaw, Poland

25 European Associated Laboratory for Gamma-Ray Astronomy, jointly supported by CNRS and MPG

26 Department of Astrophysics, Nagoya University, Chikusa-ku, Nagoya 464-8602, Japan e-mail:mori@aserv.a.phys.nagoya-u.ac.jp 
Table 3. Numerical summary of differential energy spectra for HESS J1801-233 and HESS J1800-240 (Fluxes $F$ and errors $\Delta F$ ). Results are derived using both standard and hard cuts. In cases where two flux points are extracted the weighted average flux and error (with are $99 \%$ correlated) is quoted. Statistical significances $S$ (from Li \& Ma 1983, ApJ 272, 317) from both hard-cuts and standard cuts are given when available. A minimum significance $S \geq+2 \sigma$ is required for a flux point otherwise an upper limit is quoted.

\begin{tabular}{|c|c|c|c|}
\hline Energy $E(\mathrm{TeV})$ & ${ }^{\bar{a}} F$ & ${ }^{a} \Delta F$ & $\overline{S S(\sigma)}$ \\
\hline \multicolumn{4}{|c|}{- HESS J1801-233 } \\
\hline 0.41 & 74.23 & 20.57 & $+3.7, \mathrm{NA}$ \\
\hline 0.55 & 57.40 & 10.96 & $+6.2,+5.0$ \\
\hline 0.73 & 11.81 & 4.81 & $\mathrm{NA},+2.6$ \\
\hline 0.97 & 6.51 & 2.65 & $+2.2,+3.0$ \\
\hline 1.30 & 3.11 & 1.41 & +2.3 ,NA \\
\hline 1.73 & 2.13 & 0.80 & $+2.7,+3.1$ \\
\hline 2.31 & $<3.10$ & & \\
\hline 3.08 & $<1.88$ & & \\
\hline 4.11 & 0.27 & 0.14 & $\mathrm{NA},+2.2$ \\
\hline \multicolumn{4}{|c|}{ — HESS J1800-240 A } \\
\hline 0.31 & 138.59 & 40.20 & $+3.5, \mathrm{NA}$ \\
\hline 0.41 & 87.60 & 19.41 & $+4.7, \mathrm{NA}$ \\
\hline 0.55 & 26.29 & 9.57 & $+3.6,+2.2$ \\
\hline 0.73 & 26.07 & 4.97 & $+5.8,+5.9$ \\
\hline 0.97 & 6.82 & 2.43 & $+3.3,+2.6$ \\
\hline 1.30 & 2.79 & 1.23 & $+2.5,+2.3$ \\
\hline 1.73 & 1.89 & 0.77 & $+2.6, \mathrm{NA}$ \\
\hline 2.31 & $<1.79$ & & \\
\hline 3.08 & $<1.70$ & & \\
\hline 5.48 & 0.15 & 0.08 & $\mathrm{NA},+2.2$ \\
\hline \multicolumn{4}{|c|}{- HESS J1800-240 B - } \\
\hline 0.31 & 117.92 & 40.09 & $+3.0, \mathrm{NA}$ \\
\hline 0.41 & 87.83 & 19.82 & $+4.6, \mathrm{NA}$ \\
\hline 0.55 & 38.74 & 10.24 & $+4.3,+3.7$ \\
\hline 0.73 & 17.34 & 4.80 & $+4.1,+3.6$ \\
\hline 0.97 & 6.27 & 2.49 & $+2.7, \mathrm{NA}$ \\
\hline 1.30 & 2.91 & 1.29 & $+2.6,+2.2$ \\
\hline 1.73 & 2.04 & 0.72 & $+2.9,+3.4$ \\
\hline 2.31 & 1.10 & 0.44 & $\mathrm{NA},+2.9$ \\
\hline 3.08 & $<2.25$ & & \\
\hline 4.11 & 0.27 & 0.13 & $\mathrm{NA},+2.4$ \\
\hline \multicolumn{4}{|c|}{- HESS J1800-240 C - } \\
\hline 0.55 & 19.87 & 7.62 & $+2.8, \mathrm{NA}$ \\
\hline 0.73 & 8.95 & 3.70 & $+2.6, \mathrm{NA}$ \\
\hline 1.30 & 2.68 & 1.06 & $+2.6,+3.4$ \\
\hline 1.73 & 1.09 & 0.52 & $\mathrm{NA},+2.6$ \\
\hline 2.31 & $<2.09$ & & \\
\hline 3.08 & 0.40 & 0.18 & $\mathrm{NA},+2.9$ \\
\hline \multicolumn{4}{|c|}{ - HESS J1800-240 - } \\
\hline 0.31 & 261.79 & 81.03 & $+3.3, \mathrm{NA}$ \\
\hline 0.41 & 218.26 & 39.24 & $+5.7, \mathrm{NA}$ \\
\hline 0.55 & 83.38 & 19.86 & $+3.6,+4.9$ \\
\hline 0.73 & 49.45 & 9.45 & $+5.2,+5.7$ \\
\hline 0.97 & 18.24 & 5.00 & $+3.5,+4.0$ \\
\hline 1.30 & 7.53 & 2.56 & $+2.5,+3.6$ \\
\hline 1.73 & 3.88 & 1.30 & $\mathrm{NA},+3.2$ \\
\hline 2.31 & $<6.28$ & & \\
\hline 3.08 & 1.52 & 4.37 & $\mathrm{NA},+3.8$ \\
\hline
\end{tabular}

